\title{
Andamento clinico dei soggetti eutiroidei con positività degli anticorpi anti-recettore del TSH: quanto spesso si sviluppa il morbo di Graves?
}

\author{
Nicola Viola ${ }^{1}$. Francesco Latrofa ${ }^{1}$
}

Accettato: 14 giugno 2021 / Pubblicato online: 5 ottobre 2021

(c) The Author(s) 2021

Commento a:

Clinical course of euthyroid subjects with positive TSH receptor antibody: how often does Graves' disease develop?

N. Suzuki, A. Kawaguchi, J. Yoshimura Noh,

R. Yoshimura, K. Mikura, A. Kinoshita, A. Suzuki,

T. Mitsumatsu, A. Hoshiyama, M. Fukushita,

M. Matsumoto, A. Yoshihara, N. Watanabe, K. Sugino,

K. Ito.

\section{J Endocr Soc (2021) 5(6):bvab042}

Gli anticorpi anti-recettore del TSH (TRAb) si riscontrano nel $90-100 \%$ dei pazienti affetti da morbo di Basedow. I TRAb possono essere stimolanti, inibenti o neutri e possono, pertanto, essere riscontrati anche in soggetti ipotiroidei o eutiroidei. Alcuni studi hanno dimostrato una prevalenza dei TRAb del 6,5\% in maschi eutiroidei e del $21,6 \%$ in pazienti affetti da tiroidite cronica autoimmune con eutiroidismo. Scopo di questo studio è stato quello di valutare la prevalenza dei TRAb in una coorte di donne giapponesi in età fertile ed eutiroidee e di valutare l'evoluzione clinica delle pazienti con TRAb positivi.

Lo studio, di natura retrospettiva, ha coinvolto 12.455 donne che si erano rivolte a una clinica dedicata alla fertilità, per eseguire uno screening. Dopo esclusione delle pazienti con storia di tireopatia o funzione tiroidea alterata, venivano selezionate 5.622 pazienti, nelle quale veniva valutata, oltre alla funzione, anche l'autoimmunità tiroidea mediante prelievo per AbTg, AbTPO e TRAb e veniva eseguita un'ecografia tiroidea. I prelievi FT4, FT3 e TSH e TRAb venivano

F. Latrofa

francesco.latrofa@unipi.it

1 Dipartimento di Medicina Clinica e Sperimentale, Università di Pisa, Pisa, Italia ripetuti ogni 1-6 mesi. I TRAb venivano dosati mediante immuno-assay con chemiluminescenza.

Delle 5.622 partecipanti, $47(0,84 \%)$ presentavano positività per i TRAb al tempo zero. Il follow-up (mediana: 41,6 mesi) era disponibile in 41 pazienti; di queste, $6(12,8 \%)$ sviluppavano il morbo di Basedow entro 6,6 mesi (mediana), 13 rimanevano con TRAb positivi ed eutiroidee, mentre 20 andavano incontro a negativizzazione dei TRAb. Non vi erano differenze statisticamente significative fra le pazienti che avevano sviluppato il morbo di Basedow e quelle che non lo avevano sviluppato riguardo la prevalenza degli $\mathrm{AbTg} \mathrm{e}$ degli AbTPO e il volume tiroideo.

A giudizio degli autori, questo studio dimostrerebbe che la sola positività dei TRAb non è sufficiente per lo sviluppo del morbo di Basedow, per il quale sono necessari altri fattori, genetici o ambientali. I limiti di questo studio sono rappresentati dal campione limitato a una coorte di pazienti selezionata e all'uso di un metodo di dosaggio dei TRAb che non era in grado di discriminare tra quelli stimolanti, inibenti e neutri.

Va aggiunto che i TRAb, quando positivi, lo erano a basso titolo. Questo risultato solleva il dubbio che si tratti di una falsa positività, dovuta a una bassa specificità del titolo utilizzato nello studio. La valutazione dell' attività biologica dei TRAb o, in alternativa, l'uso di un secondo kit per confermare la positività dei TRAb, sarebbero stati indubbiamente utili per rinforzare i risultati ottenuti dallo studio.

Funding Note Open access funding provided by Università di Pisa within the CRUI-CARE Agreement.

Nota della casa editrice Springer Nature rimane neutrale in riguardo alle rivendicazioni giurisdizionali nelle mappe pubblicate e nelle affiliazioni istituzionali.

Open Access This article is licensed under a Creative Commons Attribution 4.0 International License, which permits use, sharing, adaptation, distribution and reproduction in any medium or format, as long 
as you give appropriate credit to the original author(s) and the source, provide a link to the Creative Commons licence, and indicate if changes were made. The images or other third party material in this article are included in the article's Creative Commons licence, unless indicated otherwise in a credit line to the material. If material is not included in the article's Creative Commons licence and your intended use is not permitted by statutory regulation or exceeds the permitted use, you will need to obtain permission directly from the copyright holder. To view a copy of this licence, visit http://creativecommons.org/licenses/by/4.0/. 